\title{
The Near-IR Luminosity-Metallicity Relationship for Dwarf Irregular Galaxies
}

\author{
Ivo Saviane \\ European Southern Observatory, 3107 A. de Cordova, Santiago, Chile

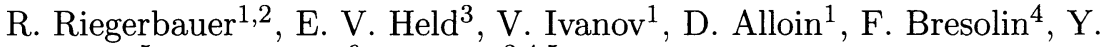 \\ Momany $^{5}$, R. M. Rich ${ }^{6}$, L. Rizzi ${ }^{3,4,5}$ \\ 1 ESO, Germany \\ ${ }^{2}$ Luleå University of Technology, Sweden \\ ${ }^{3}$ Osservatorio Astronomico di Padova, Italy \\ ${ }^{4}$ IfA Hawaii, USA \\ ${ }^{5}$ Università di Padova, Italy \\ ${ }^{6} U C L A, U S A$
}

\begin{abstract}
We briefly describe our on-going investigation of the near-IR luminosity-metallicity relationship for dwarf irregular galaxies in nearby groups of galaxies. The motivations of the project and the observational databases are introduced, and a preliminary result is presented. The $12+\log (\mathrm{O} / \mathrm{H})$ vs. $H$ plane must be populated with more low-luminosity galaxies before a definite conclusion can be drawn.
\end{abstract}

\section{The Project}

Although early studies found a very well defined luminosity-metallicity (L-Z) relationship for dwarf irregular (dIrr) galaxies (Skillman, Kennicutt, \& Hodge 1989; Richer \& McCall 1995), more recent investigations seem to suggest a mild relationship with much scatter, or no relationship at all (Hidalgo-Gamez \& Olofsson 1998; Hunter \& Hoffman 1999; Skillman, Côté, \& Miller 2003, hereafter SCM03). The main difficulties with the present status are that (a) all the studies use blue luminosities as tracers of the mass, (b) galaxies in different environments are used, (c) inhomogeneous data are used, and (d) the scatter in distance modulus is unknown. In order to overcome these limitations, we started a medium-term project whose aims are (i) collecting homogeneous samples of oxygen abundances, (ii) focusing on well-defined environments with reduced distance range, and (iii) collecting near-IR luminosities for all the objects. As a first step, we are collecting data for the three nearest groups of galaxies: the M 81 , Sculptor, and Cen A groups at a distance of about 3.5, 2.5, and 3.5 Mpc, respectively. The interaction rate in the M81 and Cen A groups is higher than that in Sculptor, so our targets offer the chance to test the effect of the environment on the L-Z relationship. 


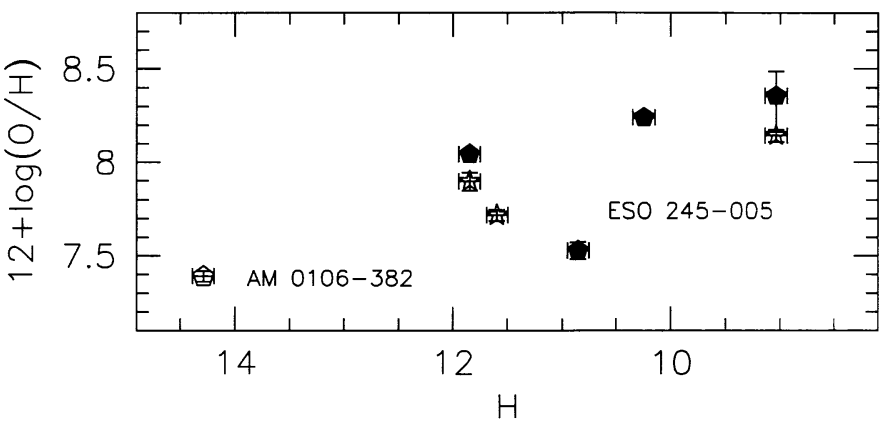

Figure 1. Oxygen abundance vs. $H$ magnitude for dIrrs of the Sculptor group in our sample (open and filled pentagons) and of that of SCM03 (stars). The open pentagon identifies an oxygen abundance computed via the empirical method.

\section{First Results}

Spectroscopy has been obtained at the Lick observatory with KAST@3.5m for the M 81 group, and at ESO with EFOSC2@3.6m for the Sculptor group. NearIR imaging has been obtained at La Palma observatory with INGRID@WHT, and at ESO with SOFI@NTT for the M81 and Sculptor groups, respectively. Observations of the Sculptor group are presented here. The reductions of the M81 group are in progress, and observations of the Cen A group have been planned.

Oxygen abundances are plotted vs. $H$ magnitudes in Fig. 1. Note that the observed HII region of ESO 245-005 lies in the outskirts of the galaxy, so the abundance of the central star-forming regions might be closer to the trend defined by the other galaxies. The trend is rather well defined, although with some scatter. On the other hand, the existence of the relationship depends very much on the single point defined by AM 106-382, whose abundance has been found via the empirical method (Edmunds \& Pagel 1984). More data are clearly needed, in particular for low-luminosity galaxies. The abundances of the objects from SCM03 agree with our determinations, reinforcing the correlation. The figure shows the advantage of working in the near-IR: the galaxies cover almost 6 magnitudes in $H$, while the SCM03 sample spans only 3 magnitudes in $B$. The impact of the unknown individual distance moduli is then reduced in the near-IR.

\section{References}

Edmunds, M.G., \& Pagel, B.E.J. 1984, MNRAS, 211, 507

Hidalgo-Gamez, A. M., \& Olofsson, K. 1998, A\&A, 334, 45

Hunter, D. A., \& Hoffman, L. 1999, AJ, 117, 2789

Richer, M.G., McCall, M.L. 1995, ApJ, 445, 642

Skillman, E.D., Kennicutt, R.C., \& Hodge, P.W. 1989, ApJ, 347, 875

Skillman, E.D., Côté, S., Miller, B.W. 2003, AJ, 125, 610 